\title{
When flocking is costly: reduced cluster-flock density over long-duration flight in pigeons
}

\author{
Daniel W. E. Sankey ${ }^{1}$ (D) $\cdot$ Steven J. Portugal ${ }^{1}$ \\ Received: 14 April 2019 /Revised: 14 April 2019 / Accepted: 25 June 2019 / Published online: 15 July 2019 \\ (C) The Author(s) 2019
}

\begin{abstract}
Birds which fly in coordinated cluster-flocks can benefit through the formation of group-level structures and patterns which can deter predators by visual confusion. Though unlike V-formation flight, cluster-flocking increases the energetic cost of flight, particularly in denser flocks. Cluster formations therefore provide a unique opportunity to investigate trade-offs between increased work rate (e.g. higher flap frequency) and other benefits of flocking. As part of a routine 9-km training flight release, a flock of six homing pigeons (Columba livia) with $5 \mathrm{~Hz}$ GPS and $200 \mathrm{~Hz}$ accelerometer biologgers attached flew an alternative trajectory totalling $177 \mathrm{~km}$ and $256 \mathrm{~min}$ of flight. We provide the first evidence that during a long-duration flight, pigeons' pairwise and group-level distances increased (i.e. group structure changed), while flap frequency decreased over time. This implies that as birds tire during long-duration flight, the ultimate functions of cluster-flocking - primarily anti-predator benefits - are overridden by the proximate costs of flying close to conspecifics.
\end{abstract}

Keywords Aerodynamics $\cdot$ Biologging $\cdot$ Collective behaviour $\cdot$ Columba livia

\section{Introduction}

The benefits for animals living in groups can include enhanced vigilance and predator detection (Elgar 1989; Parrish and Edelstein-Keshet 1999), increased foraging efficiency (Pitcher et al. 1982; Brown 1988) and the sharing of information about resources or efficient routes (Biro et al. 2006). In addition, for animals that travel in groups, potential energetic savings can be achieved through positive aero- or hydrodynamic interactions that enhance the locomotor performance of individuals in the assemblage (Portugal et al. 2014; Voelkl et al. 2015). It has been demonstrated that for birds flying in a V-formation, for example, positive aerodynamic interactions between members of the flock allow individuals to maximise

Communicated by: Matthias Waltert

Electronic supplementary material The online version of this article (https://doi.org/10.1007/s00114-019-1641-x) contains supplementary material, which is available to authorized users.

Daniel W. E. Sankey

Daniel.Sankey.2016@live.rhul.ac.uk

1 School of Biological Sciences, Royal Holloway University of London, Egham TW20 0EX, UK the capture of beneficial up-wash (Portugal et al. 2014). However, this aerodynamic power reduction due to group flight (for example, measured as a reduction in heart rate in flying white pelicans, Pelecanus onocrotalus; Weimerskirch et al. 2001) is not found in all bird groupings, with homing pigeons (Columba livia) flying in a cluster-flock having increased flap frequencies when flying near other birds (Usherwood et al. 2011). This increase in work rate is exacerbated for birds flying either at the back of the flock, or in denser formations, thus suggesting that the other aforementioned benefits of being in a group must outweigh the immediate energetic costs of cluster-flocking. Whether individuals in a flock will take measures to try and reduce the costs of flight in tight clusters - potentially by spreading out moreover long-duration flights is not currently known.

Flight is the most energetically costly form of aerobic locomotion in vertebrates, although it may be less costly in terms of energy expended per metre (i.e. the cost of transport) compared with walking or running due to the speed of travel (Alexander 2002). Nevertheless, long-duration flight is costly, and birds typically experience extensive changes in body composition before, during and after long flights (Wikelski et al. 2003). During long-duration flapping flight, it has been observed that heart rate can decrease as a function of flight time (Butler et al. 1998), assumed to be in response to the 
decrease in body mass that is observed in many species (Klaassen et al. 2000). For example, heart rate in migrating barnacle geese (Branta leucopsis) decreased by approximately $30 \%$ over the course of migration (Butler et al. 1998), while thrush nightingales (Luscinia luscinia) lost on average $3.82 \mathrm{~g}$ $(13 \%)$ of their body mass during a 12 -h flight in a wind tunnel (Klaassen et al. 2000). Thus, continuously measuring physiological and flight parameters in birds free-flying in a cluster has the potential to elucidate how individuals react to the changes in their physiology during long-duration flight, to establish if they attempt to reduce flight costs by spreading out from the cluster.

We analysed intra-group distances and flap frequency in a flock of six homing pigeons flying for 256 min over a $177-\mathrm{km}$ journey that occurred for unknown reasons during a routine 9$\mathrm{km}$ training flight. We aimed to investigate whether $(a)$ individuals begin to spread out (increased distances to nearest neighbour and the group centroid) as flight duration increases, potentially to reduce the reported effects of flying in denser flocks, and $(b)$ whether flap frequency decreases over time, to coincide with any increases in nearest neighbour distances.

\section{Materials and methods}

Six homing pigeons of unknown sex, all aged between 1 and 1.5 years (see Online Resource 1 for housing and husbandry), were transported to and released as a flock (all birds together) from a release site $9.10 \mathrm{~km}$ from the home loft, in an easterly bearing (Fig. 1; coordinates 51.46375, - 0.395744). The birds were in transit for $20 \mathrm{~min}$ in a wicker pigeon racing box $(80 \mathrm{~cm} \times 40 \mathrm{~cm} \times 22 \mathrm{~cm})$. Flight release protocol and husbandry conditions were approved by the Royal Holloway Ethical Review Board.

Each bird was tagged with both (1) a commercially available GPS logger (Qstarz BT-Q1300ST, Düsseldorf, Germany; logger mass $12 \mathrm{~g}$ ) used to measure latitude, longitude and time (GMT), five times per second (5 Hz); and (2) Axivity AX3 accelerometer (Axivity Ltd., Newcastle upon Tyne, UK) loggers (mass $8 \mathrm{~g}$ ) were used to measure three-dimensional acceleration at $200 \mathrm{~Hz}$ (Taylor et al. 2017; see Online Resource 1 for attachment protocols). The GPS units were programmed to "sports" mode, which is designed for use in fast activities involving high accelerations (up to $515 \mathrm{~ms}^{-1}$ speed, and up to 4 푔 acceleration). For full calibration procedure and accuracy testing of the GPS loggers, see Brighton et al. (2017).

The birds had been released 12 times solo and three times as a group from the site, prior to the release in question. Each bird was equipped with GPS. The birds typically returned in $15.02 \pm 41.93 \mathrm{~min}$ (median \pm s.d.) for both solo and group flights combined, with an average group route efficiency of $0.43 \pm 0.2$ (median \pm s.d.), where 1 is the beeline distance for the three group flights. The flight data for the extraordinary long flight presented took place on 18 July 2017, and the cause of the severe extended flight duration and deviation from a homeward heading is unknown. The birds were released at 13:30 BST. Upon evaluation of the GPS traces, additional weather parameters were gathered from three locations (see Online Resource 1 for full details) and assessed for repeatability to ensure dramatic changes in weather conditions were not responsible for the observed results. All parameters (wind direction, wind speed and gusts) were found to be consistent between locations (Online Resource 1).

Speed and neighbour-neighbour distance were both given by distance from one GPS position to another, using a spherical projection of the earth with radius $=63,710 \mathrm{~km}$, speed, between an individual's successive locations, and neighbourneighbour distance between two individuals at the same timestamp. Flap frequency was identified from dorsal acceleration using the upper reversal point, dorsal body amplitude by integrating dorsal acceleration twice and applying a Butterworth filter. More metrics and more detailed descriptions of our calculations and equations are provided in the Online Resource 1.

We ran three models on each of our metrics over time: (1) linear models (LM); (2) linear mixed effects models, with individual as random intercept (LME-RI); and (3) linear mixed effects models with individual as random intercept and slope (LME-RIS) (Online Resource 1). Each model was run over 1000 bootstrap iterations to calculate average AIC values for model selection. Each iteration used AEPs (autocorrelation end points; Online Resource 1) as a sampling frequency to account for autocorrelation. The model structure with the lowest mean average AIC value was chosen as the best fit for the data. One thousand iterations of the chosen model were used to estimate $R^{2}, T$ value and lower and upper confidence intervals ( $C I$; using the estimate for the fixed effect $\pm 1.96 \times$ Standard error). All statistics were calculated using $\mathrm{R}$ ( $\mathrm{R}$ core team (2017))

\section{Results and discussion}

The pigeon flock split after 97.62 min of flight. The average accuracy (the length of the trajectory divided by the beeline distance) of the group was 0.03 , taking an average of $213 \pm$ 27 min (median \pm s.d.) to return home. During the cohesive period (before the split), the nearest neighbour distance increased significantly over time (linear mixed effects model (LME); with random intercepts and slopes (RIS); $C I=0.14$, $0.57 ; R^{2}=0.34$; Figs. $2 \mathrm{a}$ and d). Similarly, individuals spread out with respect to the group centroid, in both the craniocaudal (LME-RIS; $C I=0.30,0.76 ; R^{2}=0.51$ ) and lateral (LME-RIS; $C I=0.21,0.59 ; R^{2}=0.39$ ) axes (Figs. 1d-f). Flap frequency decreased significantly over the duration of the group flight (linear model (LM); $C I=-0.23,-0.03 ; p=0.031, R^{2}=0.02$; 
a

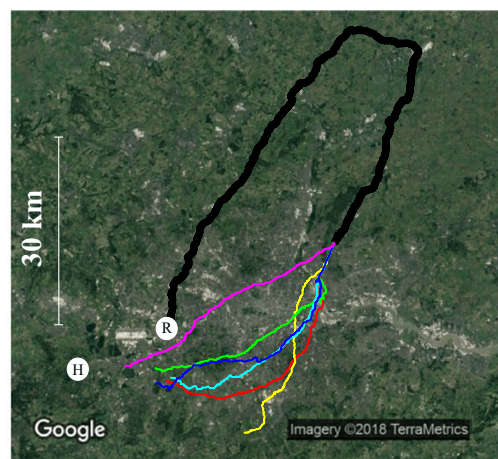

d

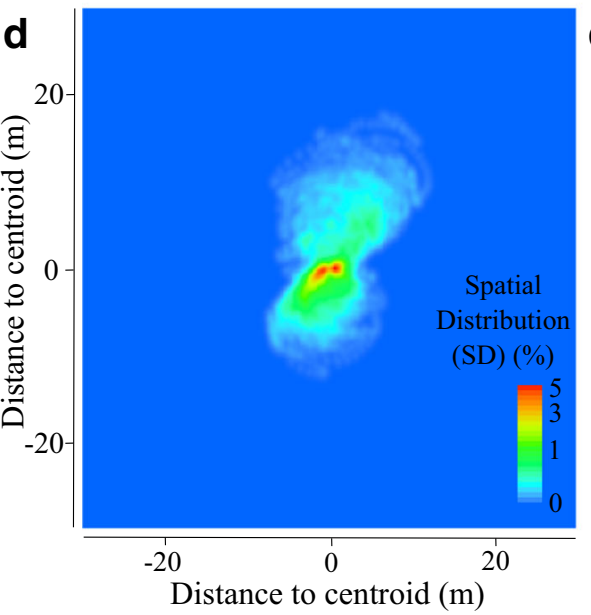

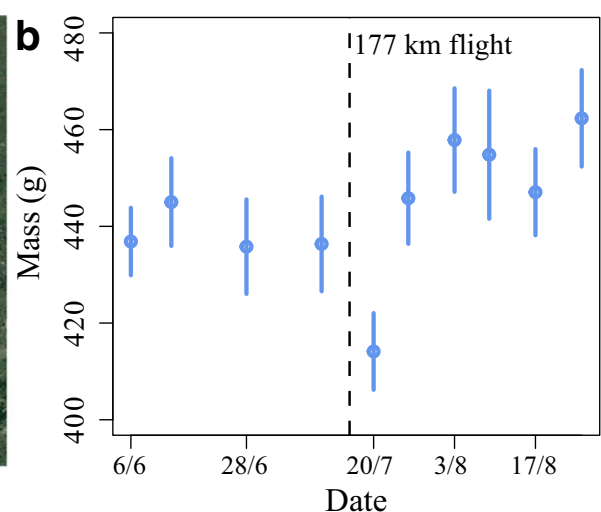

e

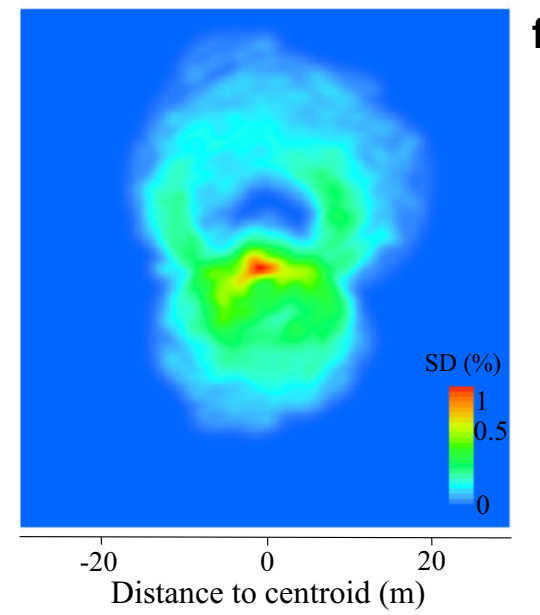

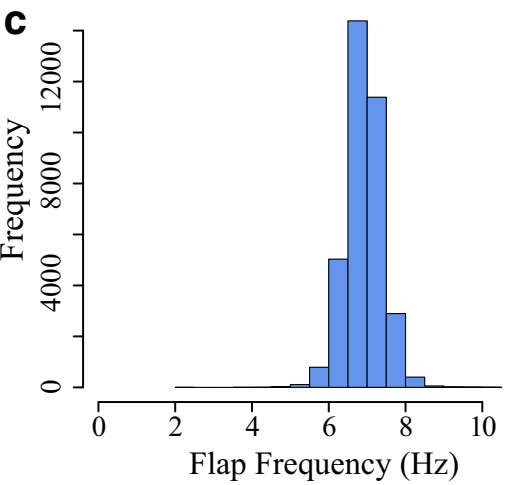

f

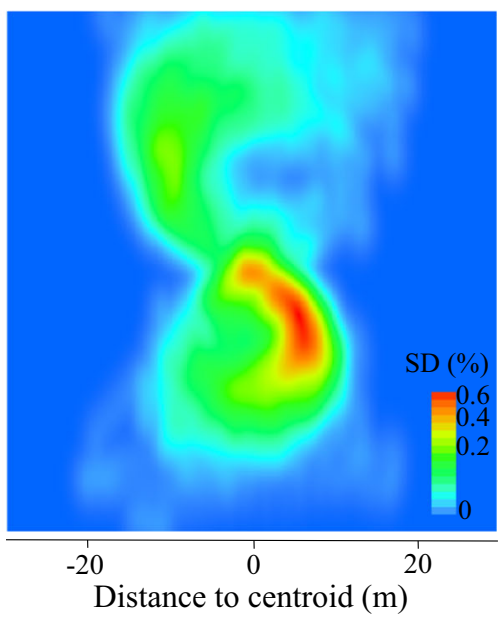

Fig. 1 Route, mass changes, flap frequency histogram and spatial structure of bird flock on long-duration flight. a Route of $177-\mathrm{km}$ flight in homing pigeons (Columba livia; $n=6$ ), plotted as black (group flight phase) and coloured (solo flight phase) lines, released from a 9.10$\mathrm{km}$ beeline distance, north-east of their home loft (white circles labelled $\mathrm{R}$ and $\mathrm{H}$ respectively), and measured via GPS data loggers. b Body mass (g) measurements of the six pigeons (mean (points) with standard error (bars)). Measurements were taken roughly weekly in the months of June, July and August. The recording following the long flight (as a categorical variable) significantly predicted a decrease in body mass ( $L M M: \mathrm{DF}=53, t=-4.30, p<0.001$ ). The $177-\mathrm{km}$ flight was recorded on 18 July (segmented line). c Frequency histogram showing accelerometer recorded flaps per second for all individuals $(n=6)$ for group phase of the flight (97.6 $\mathrm{min}$ ). d-f Frequency histogram of the group flight ( $n=6$, prior to when the birds separated) for time periods d 1-32.5 min, e 32.5$65.0 \mathrm{~min}$ and $\mathbf{f} 65.0-97.6 \mathrm{~min}$, respectively. Spatial distribution (SD) (\%) $(\mathbf{d}-\mathbf{f})$ is the estimated probability density $(\times 100)$ (using a $300 \times 300$ grid from function "stat_density2d" from package: "ggplot2" in R)
Figs. 2b and e; Online Resource 3). The low $R^{2}$ in this model is likely to be explained by the high variability in flap frequency (see Online Resource 2), though not because of interspersed flapping/gliding phases, as no gliding phases over a second long were recorded (Fig. 1c). Dorsal body amplitude did not significantly change over the duration of the cohesive flight (LME with random intercepts (RI); $C I=-0.02,0.17 ; R^{2}=$ 0.14 ; Fig. 2c). Group speed fell over the course of the cohesive period (LM; $C I=-0.53,-0.32, R^{2}=0.18$ ). It is possible that the reduction in wingbeat frequency over time was due to the birds slowing down as the flight progressed (see Online Resource 2 for full discussion on speed). Once the flock had changed their trajectory and began heading south (Fig. 1a), it is conceivable that the birds switched from a maximum range speed strategy to that of a minimum power speed (Alexander 2002). Modulation of wingbeat characteristics has previously been demonstrated to occur in response to navigational knowledge (Taylor et al. 2017), and when some appreciation of the total length of the journey is known (Lilienthal 2001). Pigeons gradually increasing their nearest neighbour distance as the flight duration increases suggest that the ultimate causes for cluster-flocking flight, such as predator confusion and increased vigilance, become outweighed by the proximate costs involved with flying close to other birds in a cluster.

As the flock began to spread out further, flap frequency decreased by approximately $0.3 \mathrm{~Hz}$. Usherwood et al. (2011) demonstrated that a tenfold increase in the spatial density of a pigeon flock was observed to be associated with a $0.1 \mathrm{~Hz}$ increase in wingbeat frequency. Similar effects have been observed more recently in flocking corvids, where flying in a group resulted in a $0.7 \mathrm{~Hz}$ increase in flap frequency versus flying solo (Ling et al. 2018). Recent work studying paired flight in homing pigeons found that individual spacing has a significant effect on wingbeat frequency over an extended 

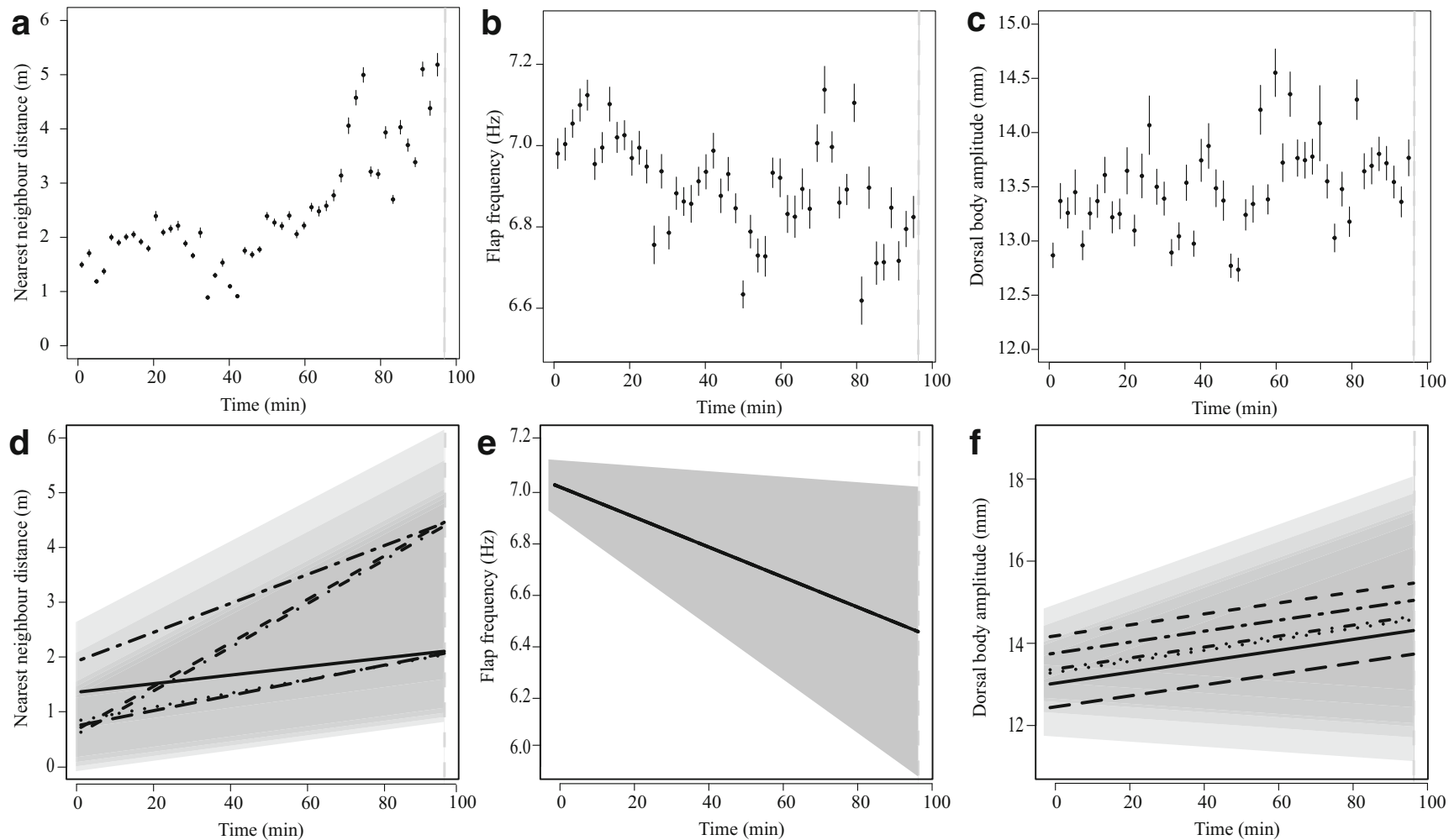

Fig. 2 Nearest neighbour distance, flap frequency and dorsal body amplitude over long-duration flight in homing pigeons (Columba livia; $n=6$ ). These three variables are plotted respectively against time, as binned means (50 bins - points; bars $=$ mean \pm s.e.m.) $(\mathbf{a}-\mathbf{c})$, and average parameters from bootstrap estimates of the best (lowest AIC) model $(\mathbf{d}-\mathbf{e})$. Dashed grey line represents where the group split (Fig. 1a). Wherever linear mixed models (LME) with individual birds as random intercepts (LME-RI) or intercept and slopes (LME-RIS) were the

range of distances, such that birds flying $50 \mathrm{~m}$ apart have an expected wingbeat frequency $0.54 \mathrm{~Hz}$ lower than those birds flying at $0 \mathrm{~m}$ spacing (Taylor et al. 2019), confirming firstly the importance of distances between individuals, but also confirming that the $\sim 0.3 \mathrm{~Hz}$ flap frequency difference identified in the present study can be considered functionally significant.

The body mass loss after the long-duration flight was $22.23 \pm 8.03 \mathrm{~g}$ (mean \pm s.d.) for the six pigeons, with the respective measurements taken 7 days before and 2 days after the extended flight (Fig. 1b). We ran a linear mixed model with body mass as a dependent variable, and the date after the long flight as a different category than other data. Pigeon ID and Date were included as random intercepts. The long flight category significantly predicted a drop-in mass by an average of $32 \mathrm{~g} \pm 7.5 \mathrm{~g}$ (s.e.m.) (LMM: DF $=53, t=-4.30, p<0.001$ ). It is possible the reduction in flap frequency was a response to the expected loss in body mass. However, if body mass loss was the primary driver for the observed decline in flap frequency (Figs. 2b and d), we would expect flap frequency to appropriate models, estimates for the intercepts and/or slopes per individual are plotted as segmented lines (d; LME-RIS, $C I=0.14,0.57)$ (f; LME-RI, $C I=-0.02,0.17)$. Fixed effects are plotted as a thick black line (d-f), and where mixed models did not yield the minimum for AIC scores, only fixed effects are plotted (e; LM, $d f=246, p=0.031)$. f Dorsal body amplitude $(\mathrm{mm})$ did not significantly change over time (using 95\% confidence intervals)

continue to decrease over the solo flight period following the split, which was not the case. This strongly suggests that it is the absence of close neighbours that prompted the reduction in flap frequency, rather than the decrease in body mass. The two components, however, do not necessarily need to be mutually exclusive.

Acknowledgements We thank the following people for useful discussions: Dora Biro, Emily Shepard, Liz Greenyer and Lucy Taylor.

Funding information Funding was provided by the Royal Society Research Grant (R10952) to S.J.P.

\section{Compliance with ethical standards}

Flight release protocol and husbandry conditions were approved by the Royal Holloway Ethical Review Board.

Open Access This article is distributed under the terms of the Creative Commons Attribution 4.0 International License (http:// creativecommons.org/licenses/by/4.0/), which permits unrestricted use, distribution, and reproduction in any medium, provided you give appropriate credit to the original author(s) and the source, provide a link to the Creative Commons license, and indicate if changes were made. 


\section{References}

Alexander RM (2002) Principles of animal locomotion. Princeton University Press, Princeton

Biro D, Sumpter DJT, Meade J, Guilford T (2006) From compromise to leadership in pigeon homing. Curr Biol 16:2123-2128. https://doi. org/10.1016/j.cub.2006.08.087

Brighton CH, Thomas ALR, Taylor GK (2017) Terminal attack trajectories of peregrine falcons are described by the proportional navigation guidance law of missiles. PNAS 114:13495-13500

Brown JS (1988) Patch use as an indicator of habitat preference, predation risk, and competition. Behav Ecol Sociobiol 22:37-47

Butler PJ, Woakes AJ, Bishop CM (1998) Behaviour and physiology of Svalbard barnacle geese Branta leucopsis during their autumn migration. J Avian Biol:536-545

Elgar MA (1989) Predator vigilance and group size in mammals and birds: a critical review of the empirical evidence. Biol Rev 64:13-33

Klaassen M, Kvist A, Lindström Å (2000) Flight costs and fuel composition of a bird migrating in a wind tunnel. Condor 102:444-451

Lilienthal O (2001) Birdflight as the basis of aviation [transl.]. Markowski International, Hummelstown

Ling H, Mclvor GE, Nagy G, MohaimenianPour S, Vaughan RT, Thornton A, Ouellette NT (2018) Simultaneous measurements of three-dimensional trajectories and wingbeat frequencies of birds in the field. J R Soc Interface 15:20180653

Parrish JK, Edelstein-Keshet L (1999) Complexity, pattern, and evolutionary trade-offs in animal aggregation. Science 284:99-101

Pitcher TJ, Magurran AE, Winfield IJ (1982) Fish in larger shoals find food faster. Behav Ecol Sociobiol 10:149-151
Portugal SJ, Hubel TY, Fritz J, Heese S, Trobe D, Voelkl B, Hailes S, Wilson AM, Usherwood JR (2014) Upwash exploitation and downwash avoidance by flap phasing in ibis formation flight. Nature 505: 399-402

R Core Team (2017) R: a language and environment for statistical computing. R Foundation for Statistical Computing, Vienna 2016

Taylor LA, Portugal SJ, Biro D (2017) Homing pigeons (Columba livia) modulate wingbeat characteristics as a function of route familiarity. $\mathrm{J}$ Exp Biol 220:2908-2915

Taylor LA, Taylor GK, Biro D, Lambert B, Walker JA, Portugal, SJ (2019) Birds invest wingbeats to keep a steady head and reap the ultimate benefits of flying together. PLOS Biol 17(6):e3000299

Usherwood JR, Stavrou M, Lowe JC, Roskilly K, Wilson AM (2011) Flying in a flock comes at a cost in pigeons. Nature 474:494-497. https://doi.org/10.1038/nature10164

Voelk1 B, Portugal SJ, Unsöld M, Usherwood JR, Wilson AM, Fritz J (2015) Matching times of leading and following suggest cooperation through direct reciprocity during V-formation flight in ibis. PNAS 112:2115-2120. https://doi.org/10.1073/pnas.1413589112

Weimerskirch H, Martin J, Clerquin Y, Alexandre P, Jiraskova S (2001) Energy saving in flight formation. Nature 413:697-698

Wikelski M, Tarlow EM, Raim A, Diehl RH, Larkin RP, Visser GH (2003) Avian metabolism: costs of migration in free-flying songbirds. Nature 423:704

Publisher's note Springer Nature remains neutral with regard to jurisdictional claims in published maps and institutional affiliations. 